\title{
Triterpenoids and Sterols from the Leaves and Twigs of Melia azedarach
}

\author{
Wei-Ming Zhang $\cdot$ Jie-Qing Liu $\cdot$ Xing-Rong Peng • \\ Luo-Sheng Wan · Zhi-Run Zhang • Zhong-Rong Li • \\ Ming-Hua Qiu
}

Received: 22 March 2014/ Accepted: 18 April 2014/Published online: 9 May 2014

(C) The Author(s) 2014. This article is published with open access at Springerlink.com

\begin{abstract}
Two new triterpenoids (1 and 2) and a new sterol (3), together with six known constituents (4-9), were isolated from the leaves and twigs of Melia azedarach. Their chemical structures were elucidated on the basis of spectroscopic analysis.
\end{abstract}

Keywords Meliaceae $\cdot$ Melia azedarach $\cdot$ Triterpenoids $\cdot$ Sterols

\section{Introduction}

Melia azedarach Linn. (Meliaceae) are widely distributed in southern districts of the Yellow River in China. The fruits and bark are commonly used as famous Traditional Chinese Medicine for acesodyne and disinsection [1]. This species has been reported to contain triterpenoids, steroids, limonoids, flavonoid glycosides, and simple phenolics [2], which have been found to possess some benefic pharmacological effects, including analgesic, anticancer, antiviral, antimalarial, antibacterial, and antifeedant activities [3, 4].

As a well known natural pesticide, azadirachtin has attracted much attention [5]. Previous investigations of the bark and roots of $M$. azedarach have shown that it is a rich

Electronic supplementary material The online version of this article (doi:10.1007/s13659-014-0019-1) contains supplementary material, which is available to authorized users.

W.-M. Zhang · J.-Q. Liu · X.-R. Peng · L.-S. Wan ·

Z.-R. Zhang · Z.-R. Li · M.-H. Qiu ( $₫)$

State Key Laboratory of Phytochemistry and Plant Resources in

West China, Kunming Institute of Botany, Chinese Academy of

Sciences, Kunming 650201, China

e-mail: mhchiu@mail.kib.ac.cn

W.-M. Zhang · M.-H. Qiu

University of Chinese Academy of Sciences, Beijing 100049,

China source of meliacarpinin type limonoids [6-10]. Until now, few chemical studies have analyzed its leaves and twigs, which prompted us to conduct this project. We identified three new compounds: a meliacarpinin type limonoid (1), an apotirucallane derivative (2), and a sterol (3), together with six known compounds (4-9) (Fig. 1). Herein, we report the details of the isolation, structural elucidation of compounds 1-3.

\section{Results and Discussion}

The air-dried powder of $M$. azedarach leaves and twigs was extracted with $\mathrm{MeOH}(30 \mathrm{~L} \times 3)$ at room temperature three times to give the residue, which was then partitioned between $\mathrm{CHCl}_{3}$ and water to get the $\mathrm{CHCl}_{3}$ soluble fraction. Then, three new constituents together with six known compounds were acquired by a series of chromatographic methods. Herein, we described the isolation and structural elucidation of these new compounds.

Compound 1 was isolated as an amorphous powder. The molecular formula was determined as $\mathrm{C}_{37} \mathrm{H}_{50} \mathrm{O}_{15}$ from the HREIMS ion peak at $\mathrm{m} / z 734.3159[\mathrm{M}]^{+}$(calcd for 734.3150). Its IR spectrum showed the presence of hydroxyl $\left(3456 \mathrm{~cm}^{-1}\right)$ and carbonyl $\left(1739 \mathrm{~cm}^{-1}\right)$ groups. The 1D NMR data (Table 1) of $\mathbf{1}$ displayed characteristic signals of meliacarpinin skeleton with three methyls $\left(\delta_{\mathrm{H}}\right.$ 


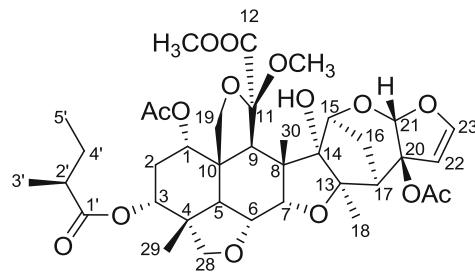

1

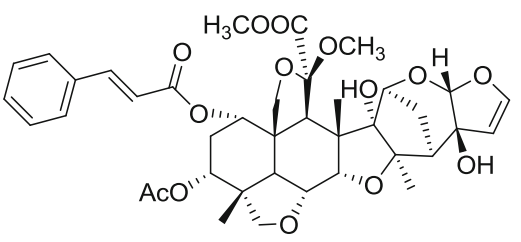

4

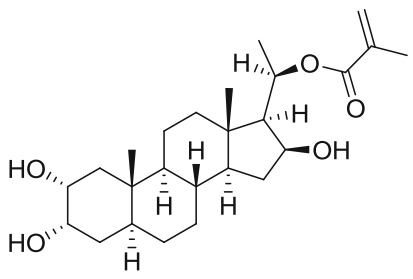

7

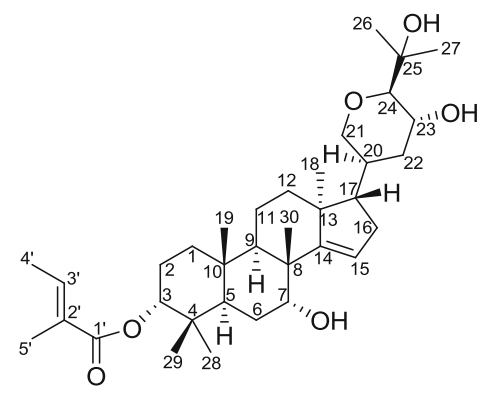

2

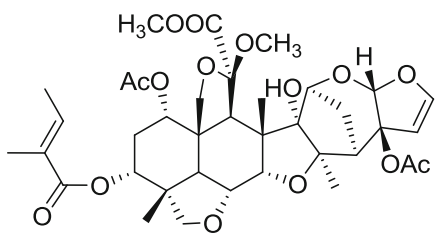

5

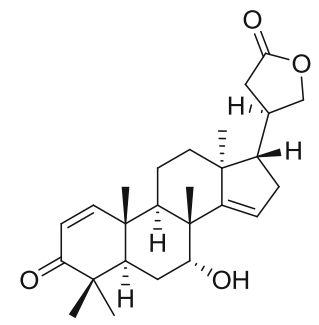

8

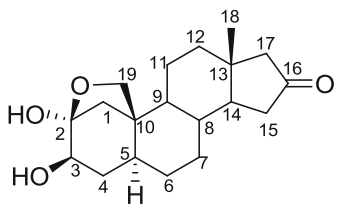

3

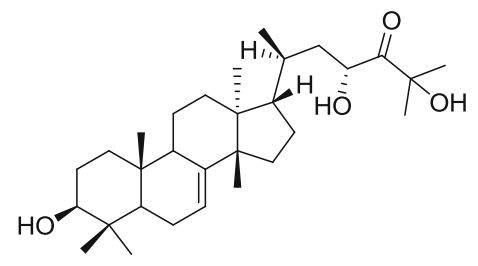

6

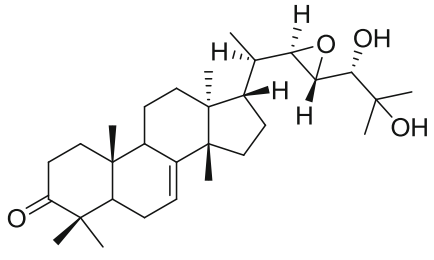

9

Fig. 1 The structures of compounds 1-9

$\left.1.75, \mathrm{~s}, 3 \mathrm{H} ; \delta_{\mathrm{H}} 0.95, \mathrm{~s}, 3 \mathrm{H} ; \delta_{\mathrm{H}} 1.66, \mathrm{~s}, 3 \mathrm{H}\right)$, two methoxyls $\left(\delta_{\mathrm{H}} 3.29, \mathrm{~s}, 3 \mathrm{H} ; \delta_{\mathrm{H}} 3.79, \mathrm{~s}, 3 \mathrm{H}\right)$, two acetyls $\left(\delta_{\mathrm{H}} 1.90, \mathrm{~s}, 3 \mathrm{H}\right.$; $\left.\delta_{\mathrm{H}} 2.30, \mathrm{~s}, 3 \mathrm{H}\right)$, one 2-methylbutyryl $\left(\delta_{\mathrm{H}} 2.59, \mathrm{~m} ; \delta_{\mathrm{H}} 1.27\right.$, $\mathrm{d}, J=7.1 \mathrm{~Hz} ; \delta_{\mathrm{H}} 1.53, \mathrm{~m} ; \delta_{\mathrm{H}} 2.02, \mathrm{~m} ; \delta_{\mathrm{H}} 0.99, \mathrm{t}$, $J=7.4 \mathrm{~Hz})$ and one hydroxyl $\left(\delta_{\mathrm{H}} 4.34, \mathrm{~s}, 1 \mathrm{H}\right)$ groups, which had a close resemblance to 3-tigloyl-1,20-diacetyl11-methoxymeliacarpinin [8], except for the presence of one 2-methylbutyryl moiety in $\mathbf{1}$ instead of the tigloyl group at C-3 in. 3-tigloyl-1,20-acetyl-11-methoxymeliacarpinin. Observed the HMBC correlations (Fig. 2) of of $\mathrm{H}-2^{\prime}\left(\delta_{\mathrm{H}} 2.59, \mathrm{~m}\right), \mathrm{H}-3^{\prime}\left(\delta_{\mathrm{H}} 1.27, \mathrm{~d}, J=7.1 \mathrm{~Hz}\right)$, $\mathrm{H}-4^{\prime} \mathrm{a}\left(\delta_{\mathrm{H}} 1.53, \mathrm{~m}\right)$ with $\mathrm{C}-1^{\prime}\left(\delta_{\mathrm{C}} 176.1\right)$, and ${ }^{1} \mathrm{H}^{1}{ }^{1} \mathrm{H}$ COSY correlations of $\mathrm{H}-3^{\prime} / \mathrm{H}-2^{\prime} / \mathrm{H}-4^{\prime} / \mathrm{H}-5^{\prime} \quad\left(\delta_{\mathrm{H}}, \quad 0.99, \quad \mathrm{t}\right.$, $J=7.4 \mathrm{~Hz}$ ) confirmed above deduction. The linkage of 2-methylbutyryl moiety to $\mathrm{C}-3$ was determined by the HMBC correlations from $\mathrm{H}-3\left(\delta_{\mathrm{H}} 4.96\right.$, br. $\left.\mathrm{t}, J=2.7 \mathrm{~Hz}\right)$ to $\mathrm{C}-1\left(\delta_{\mathrm{C}} 71.2\right), \mathrm{C}-5\left(\delta_{\mathrm{C}} 35.2\right)$, and $\mathrm{C}-1^{\prime}$.

The absolute configuration of C-2' was determined as $S$, supported by the $[\alpha]_{\mathrm{D}}^{15}$ value at +16.3 of $(S)$-2-methylbutyric acid derived from 1 by alkaline hydrolysis $\left([\alpha]_{\mathrm{D}}^{22}\right.$ -14.3 for $(R)$-2-methylbutyric acid and $[\alpha]_{\mathrm{D}}^{25}+19.3$ for (S)-2-methylbutyric acid) [11, 12]. The ROESY correlation
(Fig. 3) between $\mathrm{H}-3$ and $\mathrm{H}-6 \beta\left(\delta_{\mathrm{H}} 4.12\right.$, br. d, $J=9.2 \mathrm{~Hz}$ ) indicated that the 2-methylbutyryloxy was $\alpha$-oriented. Other relative configuration of $\mathbf{1}$ were identical with those of 3-tigloyl-1,20-acetyl-11-methoxyneliacarpinin on the basis of ROESY spectrum. Therefore, chemical structure of 1 was deduced as 3 $\alpha$-(2-methylbutyryl)- 1,20-diacetyl-11methoxymeliacarpinin.

Compound 2 was obtained as an amorphous powder. Based on the positive HREIMS $(\mathrm{m} / \mathrm{z} 572.4083$, calcd for 572.4077), the molecular formula was defined as $\mathrm{C}_{35} \mathrm{H}_{56} \mathrm{O}_{6}$. The ${ }^{1} \mathrm{H}$ NMR, ${ }^{13} \mathrm{C}$-DEPT (Table 1) spectra showed the presence of nine methyls (two of which belonged to a tigloyl), eight methylenes (one oxygenated), eight methines (four oxygenated), one trisubstituted double bond, and four quaternary carbon. These data suggested that 2 was the apo-tirucallol (euphol) skeleton [13]. Comparison of NMR data of $\mathbf{2}$ with those of compound $\mathbf{5}$ (CAS NO: 1002345-41-6) revealed that they were similar [14], except that a senecioyl ester side chain at C-3 in compound 5 was replaced by a tigloyl group $\left(\delta_{\mathrm{C}} 169.3 \mathrm{C}-1^{\prime}, 130.3\right.$ $\mathrm{C}-2^{\prime}, 138.6 \mathrm{C}-3^{\prime}, 14.6 \mathrm{C}-4^{\prime}$, and $\left.12.4 \mathrm{C}-5^{\prime}\right)$ in 2 [8], which was confirmed by the HMBC correlations (Fig. 2) of H-3 
Table $1{ }^{1} \mathrm{H}$ NMR and ${ }^{13} \mathrm{C}$ NMR spectroscopic data of $\mathbf{1}$ and $\mathbf{2}$

\begin{tabular}{|c|c|c|c|c|c|}
\hline \multirow[t]{2}{*}{ Pos } & \multicolumn{2}{|l|}{$\mathbf{1}^{\mathrm{a}}$} & \multirow[t]{2}{*}{ Pos } & \multicolumn{2}{|l|}{$2^{\mathrm{b}}$} \\
\hline & $\delta_{\mathrm{H}}(J, \mathrm{~Hz})$ & $\delta_{\mathrm{C}}$ & & $\delta_{\mathrm{H}}(J, \mathrm{~Hz})$ & $\delta_{\mathrm{C}}$ \\
\hline 1 & $4.26(\mathrm{~d}, 9.3)$ & $71.2 \mathrm{~d}$ & $1 \mathrm{a}$ & $1.27(\mathrm{~m})$ & $35.0 \mathrm{t}$ \\
\hline $2 \mathrm{a}$ & $2.27(\mathrm{~m})$ & $28.4 \mathrm{t}$ & $1 b$ & $1.43(\mathrm{~m})$ & \\
\hline $2 b$ & $2.34(\mathrm{~m})$ & & $2 \mathrm{a}$ & $1.60(\mathrm{~m})$ & $23.9 \mathrm{t}$ \\
\hline 3 & 4.96 (br. t, 2.7) & $71.6 \mathrm{~d}$ & $2 b$ & $1.99(\mathrm{~m})$ & \\
\hline 4 & & $42.9 \mathrm{~s}$ & 3 & $4.65(\mathrm{t}, 2.7)$ & $80.1 \mathrm{~d}$ \\
\hline 5 & $3.33(\mathrm{~d}, 12.7)$ & $35.2 \mathrm{~d}$ & 4 & & $37.7 \mathrm{~s}$ \\
\hline 6 & 4.12 (br. d, 9.2) & $72.1 \mathrm{~d}$ & 5 & $2.09(\mathrm{~m})$ & $43.5 \mathrm{~d}$ \\
\hline 7 & 4.53 (br. d, 5.7) & $84.0 \mathrm{~d}$ & $6 a$ & $1.71(\mathrm{~m})$ & $25.6 \mathrm{t}$ \\
\hline 8 & & $52.3 \mathrm{~s}$ & $6 b$ & $1.83(\mathrm{~m})$ & \\
\hline 9 & $3.84(\mathrm{~s})$ & $48.5 \mathrm{~d}$ & 7 & 3.95 (s-like) & $74.1 \mathrm{~d}$ \\
\hline 10 & & $50.1 \mathrm{~s}$ & 8 & & $45.3 \mathrm{~s}$ \\
\hline 11 & & $107.7 \mathrm{~s}$ & 9 & $2.12(\mathrm{~m})$ & $43.7 \mathrm{~d}$ \\
\hline 12 & & $170.5 \mathrm{~s}$ & 10 & & $38.9 \mathrm{~s}$ \\
\hline 13 & & $94.1 \mathrm{~s}$ & $11 \mathrm{a}$ & $1.53(\mathrm{~m})$ & $17.9 \mathrm{t}$ \\
\hline 14 & & $93.2 \mathrm{~s}$ & $11 b$ & $1.71(\mathrm{~m})$ & \\
\hline 15 & 4.34 (overlap) & $82.3 \mathrm{~d}$ & $12 \mathrm{a}$ & $1.55(\mathrm{~m})$ & $36.3 \mathrm{t}$ \\
\hline $16 \mathrm{a}$ & $1.93(\mathrm{~m})$ & $29.4 \mathrm{t}$ & $12 b$ & $1.93(\mathrm{~m})$ & \\
\hline $16 b$ & $2.26(\mathrm{~m})$ & & 13 & & $47.9 \mathrm{~s}$ \\
\hline 17 & $3.18(\mathrm{~d}, 5.9)$ & $48.7 \mathrm{~d}$ & 14 & & $162.7 \mathrm{~s}$ \\
\hline 18 & $1.75(\mathrm{~s})$ & $26.2 \mathrm{q}$ & 15 & $5.49(\mathrm{~d}, 2.4)$ & $121.1 \mathrm{~d}$ \\
\hline $19 \mathrm{a}$ & 4.12 (br. d, 9.2) & $70.7 \mathrm{t}$ & $16 \mathrm{a}$ & $2.12(\mathrm{~m})$ & $35.9 \mathrm{t}$ \\
\hline $19 b$ & 5.01 (overlap) & & $16 b$ & $2.31(\mathrm{ddd}, 15.1,7.3,3.6)$ & \\
\hline 20 & & $92.2 \mathrm{~s}$ & 17 & $2.04(\mathrm{~m})$ & $53.8 \mathrm{~d}$ \\
\hline 21 & $5.98(\mathrm{~s})$ & $106.7 \mathrm{~d}$ & 18 & $1.03(\mathrm{~s})$ & $19.6 \mathrm{q}$ \\
\hline 22 & $5.59(\mathrm{~d}, 3.0)$ & $106.2 \mathrm{~d}$ & 19 & $0.96(\mathrm{~s})$ & $16.1 \mathrm{q}$ \\
\hline 23 & $6.65(\mathrm{~d}, 3.0)$ & $147.6 \mathrm{~d}$ & 20 & $1.94(\mathrm{~m})$ & $37.4 \mathrm{~d}$ \\
\hline $28 \mathrm{a}$ & $3.68(\mathrm{~d}, 3.0)$ & $76.7 \mathrm{t}$ & $21 \mathrm{a}$ & $3.46(\mathrm{dd}, 11.5,2.6)$ & $71.3 \mathrm{t}$ \\
\hline $28 b$ & 3.70 (br. s) & & $21 b$ & $4.02(\mathrm{~d}, 11.5)$ & \\
\hline 29 & $0.95(\mathrm{~s})$ & $18.2 \mathrm{q}$ & $22 \mathrm{a}$ & $2.01(\mathrm{~m})$ & $37.6 \mathrm{t}$ \\
\hline 30 & $1.66(\mathrm{~s})$ & $18.5 \mathrm{q}$ & $22 b$ & $1.56(\mathrm{~m})$ & \\
\hline $14-\mathrm{OH}$ & $4.34(\mathrm{~s})$ & & 23 & $3.83(\mathrm{ddd}, 10.8,9.0,4.6)$ & $65.7 \mathrm{~d}$ \\
\hline 11-OMe & $3.29(\mathrm{~s})$ & $52.4 \mathrm{q}$ & 24 & $2.88(\mathrm{~d}, 9.0)$ & $87.8 \mathrm{~d}$ \\
\hline 12-OMe & $3.79(\mathrm{~s})$ & $53.0 \mathrm{q}$ & 25 & & $74.5 \mathrm{~s}$ \\
\hline $1-\mathrm{CH}_{3} \underline{\mathrm{CO}}$ & & $170.5 \mathrm{~s}$ & 26 & $1.22(\mathrm{~s})$ & $24.6 \mathrm{q}$ \\
\hline $20-\mathrm{CH}_{3} \underline{\mathrm{CO}}$ & & $171.2 \mathrm{~s}$ & 27 & $1.23(\mathrm{~s})$ & $28.0 \mathrm{q}$ \\
\hline $1-\underline{C}_{3} \mathrm{COO}$ & $1.90(\mathrm{~s})$ & $20.6 \mathrm{q}$ & 28 & $0.85(\mathrm{~s})$ & $28.4 \mathrm{q}$ \\
\hline $20-\underline{C H}_{3} \mathrm{COO}$ & $2.30(\mathrm{~s})$ & $21.5 \mathrm{q}$ & 29 & $0.95(\mathrm{~s})$ & $22.4 \mathrm{q}$ \\
\hline $1^{\prime}$ & & $176.1 \mathrm{~s}$ & 30 & $1.11(\mathrm{~s})$ & $28.7 \mathrm{q}$ \\
\hline $2^{\prime}$ & $2.59(\mathrm{~m})$ & $41.0 \mathrm{~d}$ & $1^{\prime}$ & & $169.3 \mathrm{~s}$ \\
\hline $3^{\prime}$ & $1.27(\mathrm{~d}, 7.1)$ & $16.7 \mathrm{q}$ & $2^{\prime}$ & & $130.3 \mathrm{~s}$ \\
\hline $4^{\prime} \mathrm{a}$ & $1.53(\mathrm{~m})$ & $26.3 \mathrm{t}$ & $3^{\prime}$ & $6.92(\mathrm{qq}, 7.1,1.4)$ & $138.6 \mathrm{~d}$ \\
\hline $4^{\prime} \mathrm{b}$ & $2.02(\mathrm{~m})$ & & $4^{\prime}$ & $1.81(\mathrm{dd}, 7.1,1.1)$ & $14.6 \mathrm{q}$ \\
\hline $5^{\prime}$ & $0.99(\mathrm{t}, 7.4)$ & $11.8 \mathrm{q}$ & $5^{\prime}$ & 1.85 (s-like) & $12.4 \mathrm{q}$ \\
\hline
\end{tabular}

${ }^{\mathrm{a}}$ Recorded in $\mathrm{C}_{5} \mathrm{D}_{5} \mathrm{~N} ;{ }^{1} \mathrm{H}$ and ${ }^{13} \mathrm{C}$ NMR recorded at $500,125 \mathrm{MHz}$

${ }^{\mathrm{b}}$ Recorded in $\mathrm{CD}_{3} \mathrm{OD} ;{ }^{1} \mathrm{H}$ and ${ }^{13} \mathrm{C}$ NMR recorded at $600,150 \mathrm{MHz}$ 
Table $2{ }^{1} \mathrm{H}$ NMR and ${ }^{13} \mathrm{C}$ NMR spectroscopic data of $\mathbf{3}$

\begin{tabular}{|c|c|c|c|c|c|}
\hline Pos & $\delta_{\mathrm{H}}(J, \mathrm{~Hz})$ & $\delta_{\mathrm{C}}$ & Pos & $\delta_{\mathrm{H}}(J, \mathrm{~Hz})$ & $\delta_{\mathrm{C}}$ \\
\hline $1 \mathrm{a}$ & $1.38(\mathrm{~d}, 11.3)$ & $44.3 \mathrm{t}$ & $11 \mathrm{a}$ & $1.34(\mathrm{~m})$ & $21.5 \mathrm{t}$ \\
\hline $1 b$ & $2.54(\mathrm{~d}, 11.3)$ & & $11 \mathrm{~b}$ & $1.58(\mathrm{~m})$ & \\
\hline 2 & & $106.3 \mathrm{~s}$ & $12 \mathrm{a}$ & $1.20(\mathrm{~m})$ & $38.2 \mathrm{t}$ \\
\hline 3 & $\begin{array}{l}4.11(\mathrm{dd}, 10.3 \text {, } \\
\quad 6.0)\end{array}$ & $74.7 \mathrm{~d}$ & $12 \mathrm{~b}$ & $1.59(\mathrm{~m})$ & \\
\hline $4 a$ & $1.73(\mathrm{~m})$ & $39.1 \mathrm{t}$ & 13 & & $39.2 \mathrm{~s}$ \\
\hline $4 \mathrm{~b}$ & $2.19(\mathrm{~m})$ & & 14 & $1.24(\mathrm{~m})$ & $51.7 \mathrm{c}$ \\
\hline 5 & 1.38 (overlap) & $43.8 \mathrm{~d}$ & $15 \mathrm{a}$ & $1.84(\mathrm{~m})$ & $39.7 \mathrm{t}$ \\
\hline $6 a$ & $1.16(\mathrm{~m})$ & $29.8 \mathrm{t}$ & $15 b$ & $\begin{array}{l}2.14(\mathrm{dd}, 17.9, \\
7.5)\end{array}$ & \\
\hline $6 \mathrm{~b}$ & $1.46(\mathrm{~m})$ & & 16 & & $217.5 \mathrm{~s}$ \\
\hline $7 \mathrm{a}$ & 0.79 (overlap) & $32.3 \mathrm{t}$ & $17 \mathrm{a}$ & $1.93(\mathrm{~d}, 16.6)$ & $56.2 \mathrm{t}$ \\
\hline $7 b$ & 1.37 (overlap) & & $17 \mathrm{~b}$ & $2.06(\mathrm{~d}, 16.6)$ & \\
\hline 8 & 0.80 (overlap) & $36.8 \mathrm{~d}$ & 18 & $0.64(\mathrm{~s})$ & $18.1 \mathrm{c}$ \\
\hline 9 & $1.05(\mathrm{~m})$ & $46.4 \mathrm{~d}$ & $19 \mathrm{a}$ & $3.86(\mathrm{~d}, 8.1)$ & 67.6 \\
\hline 10 & & $48.2 \mathrm{~s}$ & $19 \mathrm{~b}$ & $4.08(\mathrm{~d}, 8.1)$ & \\
\hline
\end{tabular}

Recorded in $\mathrm{C}_{5} \mathrm{D}_{5} \mathrm{~N} ;{ }^{1} \mathrm{H}$ and ${ }^{13} \mathrm{C}$ NMR recorded at $600,150 \mathrm{MHz}$

$\left(\delta_{\mathrm{H}} 4.65, \mathrm{t}, J=2.7 \mathrm{~Hz}\right), \mathrm{H}-3^{\prime}\left(\delta_{\mathrm{H}} 6.92, \mathrm{qq}, J=7.1\right.$, $1.4 \mathrm{~Hz}$ ), and $\mathrm{H}-5^{\prime}\left(\delta_{\mathrm{H}} 1.85\right.$, s-like) with $\mathrm{C}-1^{\prime}$, and of $\mathrm{H}-4^{\prime}$ $\left(\delta_{\mathrm{H}} 1.81, \mathrm{dd}, J=7.1,1.1 \mathrm{~Hz}\right)$ with $\mathrm{C}-2^{\prime}$, together with the ${ }^{1} \mathrm{H}-{ }^{1} \mathrm{H}$ COSY correlations of $\mathrm{H}-3^{\prime} / \mathrm{H}-4^{\prime}$.
The ROESY correlation (Fig. 3) between $\mathrm{H}-3$ and Me$19 \beta$ suggested that the tigloyl group at C- 3 was $\alpha$-oriented. The coupling constant between $\mathrm{H}-23$ and $\mathrm{H}-24$ $(J=9.0 \mathrm{~Hz})$ suggested their anti-periplanar relation [14], and combination with the ROESY correlations of $\mathrm{H}-17 / \mathrm{H}-$ $23, \mathrm{H}-17 / \mathrm{H}-19 \beta, \mathrm{H}-20 / \mathrm{Me}-18 \alpha$ and $\mathrm{H}-24 / \mathrm{Me}-18 \alpha$ revealed that the configuration of $\mathrm{C}-23$ and $\mathrm{C}-24$ were both $R^{*}$. Thus, the structure of 2 was established as $3 \alpha$-tigloyl-17 $\alpha$ 20S-21,24-epoxy-apotirucall-14-en-7 $\alpha, 23 \alpha, 25$-triol.

Compound $\mathbf{3}$ was isolated as an amorphous powder. The HREIMS of 3 gave a $[\mathrm{M}]^{+}$ion peak at $m / z, 320.1985$ (calcd for 320.1988), consistent with the molecular formula of $\mathrm{C}_{19} \mathrm{H}_{28} \mathrm{O}_{4}$. Detailed analysis of its ${ }^{1} \mathrm{H}$ and ${ }^{13} \mathrm{C}$-DEPT (Table 2) and 2D NMR data indicated that 3 and $2 \alpha, 3 \alpha-$ dihydroxyandrostan-16-one $2 \beta, 19$-hemiketal [15] had the same planar structure. The only difference between them was the configuration of substituent group at C-3. Comparison its ${ }^{1} \mathrm{H}$ NMR data with that of epi-isomer showed that the coupling constants of $\mathrm{H}-3\left(\delta_{\mathrm{H}} 4.11, \mathrm{dd}, J=10.3\right.$, $6.0 \mathrm{~Hz})$ and the chemical shifts for $\mathrm{H}-1 \alpha\left(\delta_{\mathrm{H}} 1.38\right.$, d, $J=11.3 \mathrm{~Hz})$ and $\mathrm{H}-1 \beta\left(\delta_{\mathrm{H}} 2.54, \mathrm{~d}, J=11.3 \mathrm{~Hz}\right)$ were obviously different from those of $2 \alpha, 3 \alpha$-dihydroxyandrostan-16-one $2 \beta, 19$-hemiketa. But the aforementioned data was familiar with $2 \alpha, 3 \beta$-dihydroxypregnan-16-one $2 \beta, 19$ hemiketal [10], which implied that the H-3 of 3 was $\alpha$ -
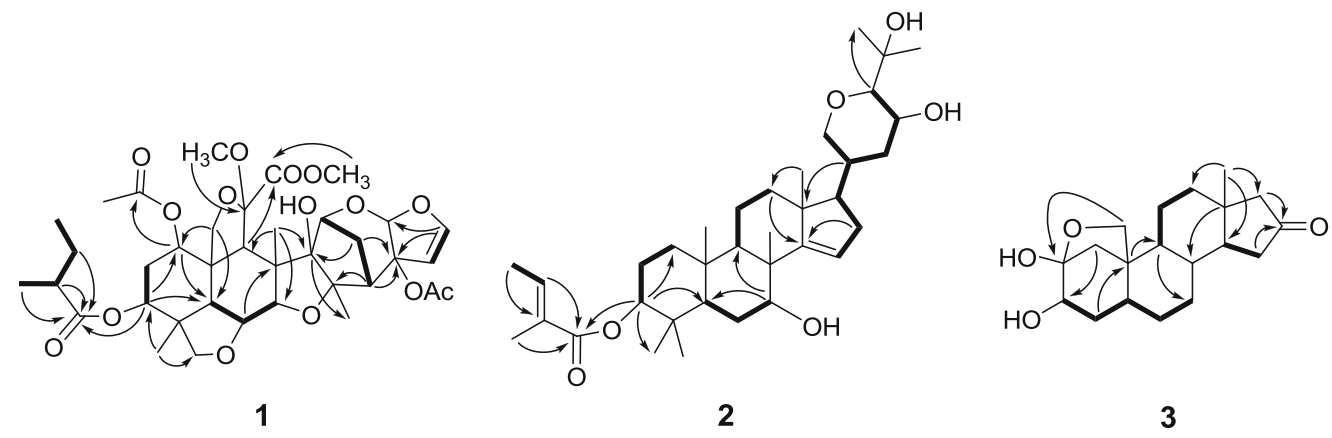

3

Fig. 2 Selected ${ }^{1} \mathrm{H}^{-1} \mathrm{H}$ COSY $(\longrightarrow)$ and $\mathrm{HMBC}(\longrightarrow)$ correlations of $\mathbf{1}-\mathbf{3}$

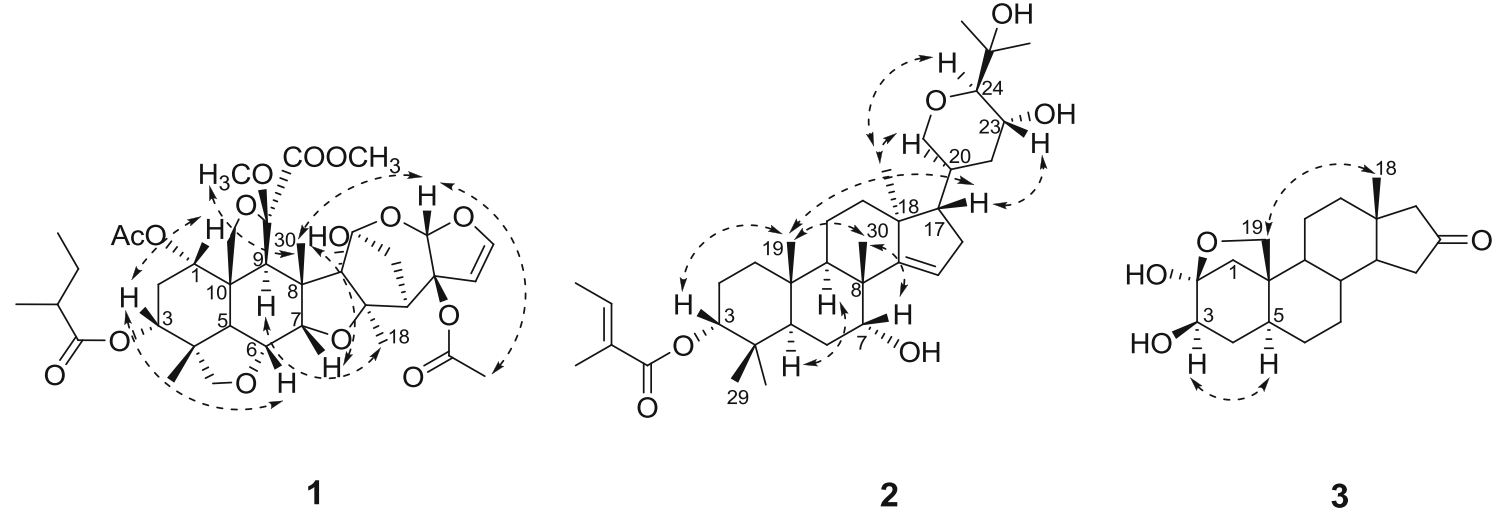

Fig. 3 Selected ROESY $(\hookrightarrow-\rightarrow)$ correlations of 1-3 
oriented. This conclusion further confirmed by the cross peak between $\mathrm{H}-3$ and $\mathrm{H}-5\left(\delta_{\mathrm{H}} 1.38\right.$, overlap) in the ROESY spectrum (Fig. 3). So the hydroxyl group at C-3 was $\beta$-configuration. Consequently, the chemical structure of $\mathbf{3}$ was elucidated as $2 \alpha, 3 \beta$-dihydroxyandrostan-16-one $2 \beta, 19$ hemiketal.

Six known constituents: 1-cinnamoyl-3-acetyl-11methoxymeliacarpinin (4) [8], 3-tigloyl-1,20-diacetyl-11methoxymeliacarpinin (5) [8], 3S,23R,25-trihydroxytirucall-7-en-24-one (6) [16], and $2 \alpha, 3 \alpha, 16 \beta$-trihydroxy- $5 \alpha-$ pregnane 20R-methacrylate (7) [17], 6-de(acetyloxy)-7deacetylchisocheton compound E (8) [18], Toonapubesin C (9) [19], were identified by comparison of their spectroscopic data with those reported in the literature.

\section{Experimental}

\subsection{General Experimental Procedures}

Optical rotations were measured with a Horiba SEPA-300 polarimeter. UV spectra were detected on a Shimadzu UV2401A spectrophotometer. IR spectra were measured on a Bruker Tensor-27 infrared spectrophotometer with $\mathrm{KBr}$ pellets. ESIMS analysis were recorded on an API QSTAR Pulsar I spectrometer. EIMS and HREIMS were performed on a Waters Autospec Premier P776 mass spectrometer. 1D and 2D NMR spectra were recorded on Bruker DRX-500 and Bruker Avance III-600 spectrometers with TMS as internal standard. Semi-preparative HPLC studies were carried out on an Agilent 1100 liquid chromatograph with a Zorbax SB-C18 $(9.4 \mathrm{~mm} \times 25 \mathrm{~cm})$ column. Column chromatography was performed with silica gel (200-300 mesh, Qingdao Marine Chemical, Inc.), Sephadex LH-20 (20-150 $\mu \mathrm{m}$, Pharmacia), and Lichroprep RP-18 (40-63 $\mu \mathrm{m}$, Merck). Fractions were monitored by TLC, and spots were visualized by heating the silica gel plates sprayed with $10 \% \mathrm{H}_{2} \mathrm{SO}_{4}$ in $\mathrm{EtOH}$.

\subsection{Plant Material}

The leaves and twigs of M. azedarach were collected from Kunming, Yunnan Province, China. A voucher sample (NO: 2011-05-07) has been deposited in the State Key Laboratory of Phytochemistry and Plant Resources in West China, Kunming Institute of Botany, Chinese Academy of Sciences.

\subsection{Extraction and Isolation}

The air-dried and powdered leaves and twigs of M. azedarach $(10 \mathrm{~kg})$ were extracted with $\mathrm{MeOH}(30 \mathrm{~L} \times 3)$ at room temperature. Evaporation of the solvent under reduced pressure provide a dark residue (700 g), which was suspended in water and then partitioned with $\mathrm{CHCl}_{3}$ and $n-\mathrm{BuOH}$, successively, to yield $\mathrm{CHCl}_{3}$ fraction $(120 \mathrm{~g}), n$-BuOH fraction $(156 \mathrm{~g})$. The $\mathrm{CHCl}_{3}$ extract was chromatographed by silica gel column eluted with $\mathrm{CHCl}_{3}-\mathrm{MeOH}$ as a gradient $(100: 1,50: 1,20: 1,5: 1)$ to afford four fractions. The $\mathrm{CHCl}_{3}-\mathrm{MeOH}(100: 1)$ portion was evaporated to obtain a residue $(20 \mathrm{~g})$, which was subjected to silica gel chromatograph column with petroleum etherEtOAc (10:1, 6:1, 3:1, 1:1) as elution, to give four fractions (A, $\mathrm{B}, \mathrm{C}$, and D). Fraction B ( $5 \mathrm{~g}$ ) was further subjected to RP-18 chromatograph column, eluting with $\mathrm{MeOH}-\mathrm{H}_{2} \mathrm{O}$ (40:60, 60:40, 80:20, and 100:0) to afford five fractions: B1-B5. Fraction B4 was then purified by HPLC $\left(70 \% \mathrm{CH}_{3} \mathrm{CN}\right.$ aq.; $2.0 \mathrm{~mL} / \mathrm{min} ; 210 \mathrm{~nm}$; Zorbax SB-C18, $9.4 \mathrm{~mm} \times 25 \mathrm{~cm}$ ) to give compounds $1(4 \mathrm{mg}), \mathbf{4}(2 \mathrm{mg})$ and $\mathbf{5}(3 \mathrm{mg})$. In the same way, $2(4 \mathrm{mg}), 6(5 \mathrm{mg})$ and $9(7 \mathrm{mg})$ were islated from fraction B3. Fraction B2 was subjected to silica gel chromatograph column with petroleum ether-EtOAc $(8: 1,5: 1,3: 1$, $1: 1$, and $0: 1)$ as elution, to give five subfractions $(\mathrm{E}, \mathrm{F}, \mathrm{G}$, and $\mathrm{H})$. Subfraction $\mathrm{F}$ was further separated and purified by silica gel chromatography column with $\mathrm{CHCl}_{3}-\mathrm{Me}_{2} \mathrm{CO}$ (50:1, 20:1, 5:1, and 1:1) as elution, get four subfraction: F1-F4, subfraction F2 was successively subjected to Sephadex LH-20 $(\mathrm{MeOH})$ and $\mathrm{HPLC}\left(80 \% \mathrm{CH}_{3} \mathrm{CN}\right.$ aq.; $2.0 \mathrm{~mL} / \mathrm{min} ; 210 \mathrm{~nm}$; Zorbax SB-C18, $9.4 \mathrm{~mm} \times 25 \mathrm{~cm})$, and compounds 3 $(1.5 \mathrm{mg}), \mathbf{7}(3 \mathrm{mg})$ and $\mathbf{8}(6 \mathrm{mg})$ were obtained.

\section{$3.43 \alpha$-(2-Methylbutyryl)-1,20-diacetyl-11- methoxymeliacarpinin (1)}

Amorphous powder; $[\alpha]_{\mathrm{D}}^{17}-17.8$ ( c $\left.0.08, \mathrm{MeOH}\right)$; UV $(\mathrm{MeOH}) \lambda_{\max }(\log \varepsilon) 208(4.09) \mathrm{nm}$; IR $(\mathrm{KBr}) v_{\max }$ 3456, 2953, 1739, 1706, 1618, 1438, 1376, 1252, 1160, 1131, 1061, and $949 \mathrm{~cm}^{-1}$; ${ }^{1} \mathrm{H}$ NMR $\left(500 \mathrm{MHz}, \mathrm{C}_{5} \mathrm{D}_{5} \mathrm{~N}\right)$ and ${ }^{13} \mathrm{C}$ DEPT (125 MHz, $\left.\mathrm{C}_{5} \mathrm{D}_{5} \mathrm{~N}\right)$ data, see Tables 1 and 2; posi-

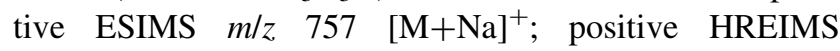
$m / z 734.3159$ (calcd for $\mathrm{C}_{37} \mathrm{H}_{50} \mathrm{O}_{15}[\mathrm{M}]^{+}, 734.3150$ ).

\section{$3.53 \alpha$-Tigloyl-17 $\alpha$-20S-21,24-epoxy-apotirucall-14- en-7 $\alpha, 23 \alpha, 25$-triol (2)}

Amorphous powder; $[\alpha]_{\mathrm{D}}^{17}-28.9$ (c $\left.0.20, \mathrm{MeOH}\right)$; UV $(\mathrm{MeOH}) \lambda_{\max }(\log \varepsilon) 204(3.80) \mathrm{nm}$; IR (KBr) $v_{\max } 3441$, 2927, 2855, 1631, 1452, 1384, 1268, 1075 and $578 \mathrm{~cm}^{-1}$; ${ }^{1} \mathrm{H}$ NMR $\left(600 \mathrm{MHz}, \mathrm{CD}_{3} \mathrm{OD}\right)$ and ${ }^{13} \mathrm{C}$ DEPT $(150 \mathrm{MHz}$, $\mathrm{CD}_{3} \mathrm{OD}$ ) data, see Tables 1 and 2; positive ESIMS $\mathrm{m} / z 595$ $[\mathrm{M}+\mathrm{Na}]^{+}$; positive HREIMS $\mathrm{m} / \mathrm{z} 572.4083$ (calcd for $\mathrm{C}_{35} \mathrm{H}_{56} \mathrm{O}_{6}[\mathrm{M}]^{+}$, 572.4077).

\subsection{2 $2 \alpha, 3 \beta$-Dihydroxyandrostan-16-one $2 \beta, 19$ -} hemiketal (3)

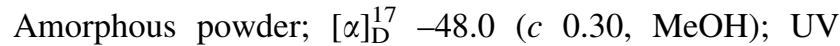
$(\mathrm{MeOH}) \lambda_{\max }(\log \varepsilon) 202(3.56), 219(3.51) \mathrm{nm}$; IR (KBr) 
$v_{\max }$ 3464, 2924, 2874, 1720, 1447, 1295, 1187, 1130, 1044, and $993 \mathrm{~cm}^{-1}$; ${ }^{1} \mathrm{H}$ NMR (600 MHz, $\left.\mathrm{C}_{5} \mathrm{D}_{5} \mathrm{~N}\right)$ and ${ }^{13} \mathrm{C}$ DEPT (150 MHz, $\left.\mathrm{C}_{5} \mathrm{D}_{5} \mathrm{~N}\right)$ data, see Tables 1 and 2; positive ESIMS $\mathrm{m} / \mathrm{z} 343[\mathrm{M}+\mathrm{Na}]^{+}$; positive HREIMS $m / z 320.1985$ (calcd for $\mathrm{C}_{20} \mathrm{H}_{28} \mathrm{O}_{5}[\mathrm{M}]^{+}, 320.1988$ ).

Acknowledgments This work was supported financially by Joint Fund of NSFC and NSFY (No.U1132604), Key Program of MOST of CHINA (Nos. 2007BAD32B03 and SB2007FY400), as well as Foundation of State Key Laboratory of Phytochemistry and Plant Resources in West China (P2010-ZZ14).

Conflict of interest The authors declare no conflict of interest.

Open Access This article is distributed under the terms of the Creative Commons Attribution License which permits any use, distribution, and reproduction in any medium, provided the original author(s) and the source are credited.

\section{References}

1. Editorial Committee of Flora of China, Chinese Academy of Science. In Flora of China, vol 43 (Science Press, Beijing, 1997), pp. 100-102

2. Z.S. Su, S.P. Yang, S. Zhang, L. Dong, J.M. Yue, Helv. Chim. Acta 94, 1515-1526 (2011)
3. S.B. Mahato, N.P. Sahu, G. Podder, Sci. Cult. 53, 29 (1987)

4. Vishnukanta, A.C. Rana, [Vishnukanta, 2008 \#2350]

5. E.D. Morgan, Bioorg. Med. Chem. 17, 4096-4105 (2009)

6. M. Nakatani, R.C. Huang, H. Okamura, T. Iwagawa, Chem. Lett. 12, 2125-2128 (1993)

7. M. Nakatani, S. Arikawa, H. Okamura, T. Iwagawa, Heterocycles 38, 327-331 (1994)

8. K. Takeya, Z.S. Qiao, C. Hirobe, H. Itokawa, Phytochemistry 42, 709-712 (1996)

9. Q.G. Tan, X.N. Li, H. Chen, T. Feng, X.H. Cai, X.D. Luo, J. Nat. Prod. 73, 693-697 (2010)

10. F. Yoshiyasu, O. Mari, T. Hironobu, M. Hiroyuki, Chem. Pharm. Bull. 48, 301-303 (2000)

11. A.I. Meyers, G. Knaus, K. Kamata, J. Am. Chem. Soc. 96, 268-270 (1974)

12. B.B. Sylvia, B. George, M. George, J. Org. Chem. 32, 2641-2642 (1967)

13. X.D. Luo, S.H. Wu, D.G. Wu, Y.B. Ma, S.H. Qi, Tetrahedron 58, 6691-6695 (2002)

14. K. Mitsui, H. Saito, R. Yamamura, H. Fukaya, Y. Hitotsuyanagi, K. Takeya, Chem. Pharm. Bull. 55, 1442-1447 (2007)

15. M.T. Pupo, P.C. Vieira, J.B. Fernandes, M.F.G.F. da Silva, E. Rodrigues Filho, Phytochemistry 45, 1495-1500 (1997)

16. M. Arisawa, A. Fujita, N. Morita, P.J. Cox, R.A. Howie, G.A. Cordell, Phytochemistry 26, 3301-3303 (1987)

17. M. Nakatani, H. Takao, I. Miura, T. Hase, Phytochemistry 24, 1945-1948 (1985)

18. J. Li, M.Y. Li, T. Satyanandamurty, J. Wu, Helv. Chim. Acta 94, 1651-1656 (2011)

19. J.R. Wang, H.L. Liu, T. Kurtan, A. Mandi, S. Antus, J. Li, H.Y. Zhang, Y.W. Guo, Org. Biomol. Chem. 9, 7685-7696 (2011) 